\title{
Untriggered search for rapid optical transients with Mini-MegaTORTORA wide-field monitoring system
}

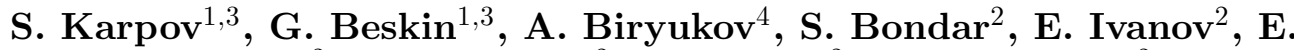 \\ Katkova $^{2}$, N. Orekhova ${ }^{2}$, A. Perkov ${ }^{2}$ and V. Sasyuk ${ }^{2}$ \\ ${ }^{1}$ Special Astrophysical Observatory of Russian Academy of Sciences, Russia. \\ ${ }^{2}$ Research and Production Corporation "Precision Systems and Instruments", Russia. \\ ${ }^{3}$ Kazan Federal University, Russia. \\ ${ }^{4}$ Moscow State University, Russia.
}

\begin{abstract}
Here we report ongoing efforts for an untriggered search of rapid optical transients of various astrophysical and non-astrophysical origins on time scales down to fractions of a second with Mini-MegaTORTORA. Mini-MegaTORTORA is a novel 9-channel wide-field optical monitoring system in operation since 2014 at Special Astrophysical Observatory on Russian Caucasus.
\end{abstract}

Keywords. gamma rays: bursts, instrumentation: miscellaneous

Mini-MegaTORTORA (Biryukov et al.2015 and references therein) is a novel robotic instrument developed to detect and characterize fast optical transients of various origins, both cosmological, galactic and near-Earth. It is a 9 -channel wide-field $(\sim 900$ square degrees) monitoring system with temporal resolution of 0.1 seconds and limit down to V 11 mag. Every channel of Mini-MegaTORTORA has 10x10 deg field of view and is equipped with installable photometric and polarimetric filters and coelostat mirror for a rapid repointing in a limited range. It allows to re-configure the system on the fly in order to rapidly follow-up the transients just detected.

Mini-MegaTORTORA started its operation in Jun 2014, and since then continuously monitors the sky looking for fast optical transients. Its real-time transient detection system routinely extracts various kinds of transient from the data stream - rapid flashes, meteors, satellites etc. Data on detected transients are published online.

Transient detection software matches rapid flashes with stellar catalogues to exclude scintillation effects and with NORAD database of satellite orbits to filter out satellite flashes. Some fraction of detected flashes are not identified with satellites, but most probably are of satellite origin. Immediate follow-up observations using Mini-MegaTORTORA rapid reaction mode (see Figure 1) typically reveal faint satellite trails. Durations and shapes of such flashes (Figure 2) are also consistent with satellite ones. Therefore we may conclude that no bright rapid flashes of astrophysical origin are detected in 2.5 years of Mini-MegaTORTORA operation.

Mini-MegaTORTORA also performs follow-up of Swift, Fermi and LIGO-Virgo triggers, including the ones with poor localization accuracy due to its large field of view allowing for simultaneous observations in $\sim 900$ sq.deg. sky fields. For the triggers with better localizations, multicolor and/or polarimetric follow-up is performed. Since mid2015, 4 of 89 Swift GRBs have been followed up in polarimetric mode in 30 to 60 seconds since trigger distribution through GCN network, with no optical emission detections. 9 of 250 Fermi GBM triggers have been also followed up in wide-field mode in 20 to 
Mini-MegaTORTORA trigger 10728736 at 2016-10-21 23:48:09
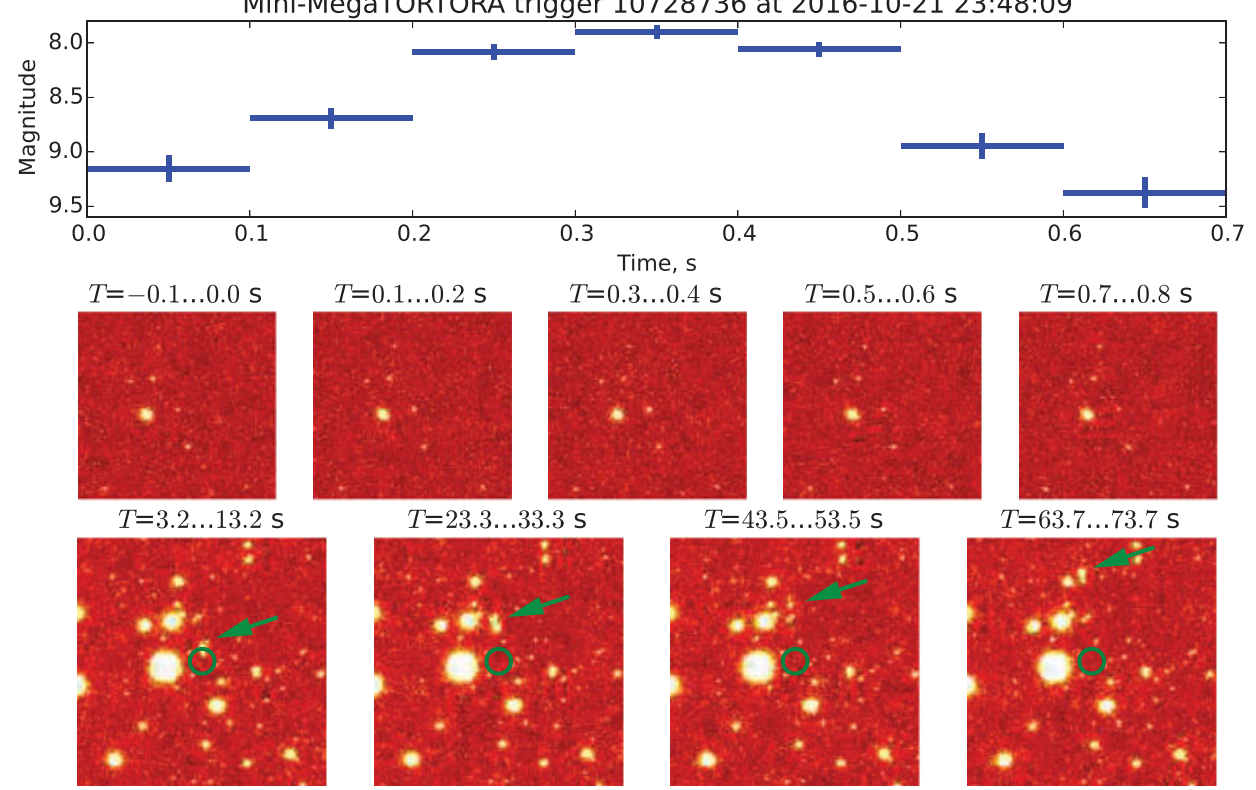

Figure 1. Example of a rapid optical flash independently detected and followed-up by Mini-MegaTORTORA and not identified with satellites from NORAD database. Upper panel light curve with $0.1 \mathrm{~s}$ temporal resolution, middle panel - corresponding detection images $\left(50^{\prime}\right.$ x $50^{\prime}$ around the event), lower panel - follow-up images with $10 \mathrm{~s}$ exposures that clearly reveal the satellite, marked by arrow, slowly moving away from the flash position, marked by circle.
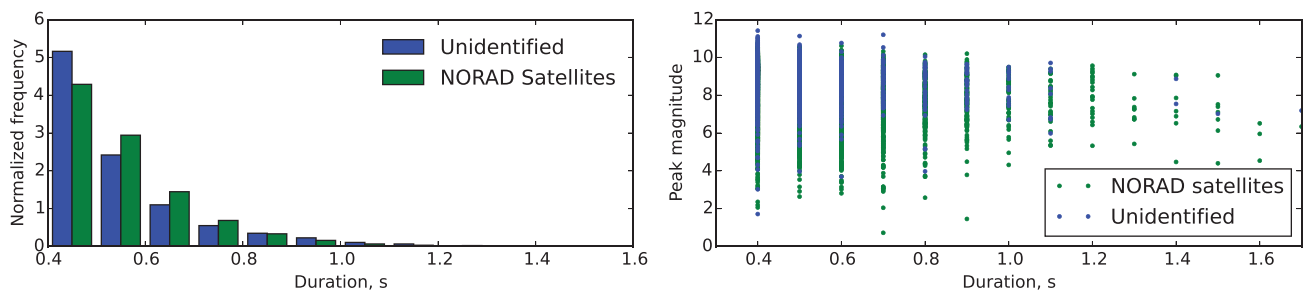

Figure 2. Comparison of durations (left panel) and brightness (right panel) of rapid flashes detected by Mini-MegaTORTORA and identified/non-indentified with NORAD satellites.

90 seconds from the trigger, with bright optical flash of GRB 160625B clearly detected during the gamma activity. All other events were either below the horizon or occurred in bad weather conditions.

\section{Acknowledgements}

Mini-MegaTORTORA belongs to Kazan Federal University and the work is performed according to the Russian Government Program of Competitive Growth of Kazan Federal University. Observations on Mini-MegaTORTORA are supported by the Russian Science Foundation grant No. 14-50-00043.

\section{Reference}

Biryukov, A., Beskin, G., Karpov, S., Bondar, S., Ivanov, E., Katkova, E., Perkov, A., \& Sasyuk, V. 2015, Baltic Astronomy, 24, 100 